\title{
Calculation of stochastic thermal transport due to resonant magnetic perturbations in DIII-D
}

\author{
I. Joseph ${ }^{\mathrm{a}, \mathrm{b}}$ *, T.E. Evans ${ }^{\mathrm{b}}$, A.M. Runov ${ }^{\mathrm{c}}$, M.E. Fenstermacherd ${ }^{\mathrm{d}}$, M. Groth ${ }^{\mathrm{d}}$, \\ S.V. Kasilove,f, C.J. Lasnierd, R.A. Moyera, G.D. Porterd, M.J. Schaffer ${ }^{\text {b }}$, \\ R. Schneiderc, and J.G. Watkins g
}

aUniversity of California-San Diego, 9500 Gilman Dr., La Jolla, California, USA

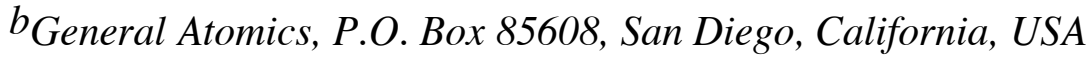

${ }^{c}$ Max Planck Institut für Plasmaphysik, EURATOM Association, Teilinstitut Greifswald, Greifswald, Germany

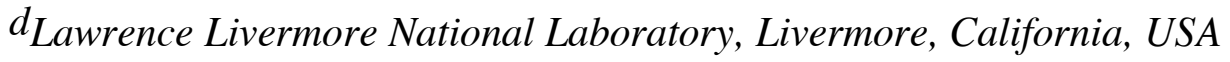

eInstitute of Plasma Physics, National Science Center "Kharkov Institute of Physics and Technology," Kharkov, Ukraine

f Association EURATOM-OEAW, Institute für Theoretische Physik-Computational Physics, Technische Universität Graz, Graz, Austria

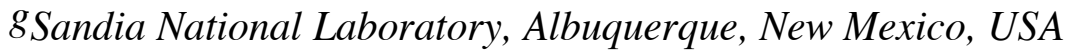

*Corresponding author: I. Joseph, General Atomics, P.O. Box 85608, MS 13-368, San Diego, California 92186-5608,e-mail: ijoseph@ucsd.edu

\begin{abstract}
The effect of resonant magnetic perturbations on heat transport in DIII-D H-mode plasmas has been calculated by combining the TRIP3D field-line tracing code with the E3D two-fluid transport code. Simulations show that the divertor heat flux distribution becomes non-axisymmetric because heat flux is efficiently guided to the
\end{abstract}


divertor along the three-dimensional invariant manifolds of the magnetic field. Calculations demonstrate that heat flux is spread over a wider area of the divertor target, thereby reducing the peak heat flux delivered during steady-state operation. Filtered optical cameras have observed non-axisymmetric particle fluxes at the strike-point and Langmuir probes have observed non-axisymmetric floating potentials. On the other hand, the predicted magnitude of stochastic thermal transport is too large to match the pedestal plasma profiles measured by Thomson scattering and charge exchange recombination spectroscopy. The Braginskii thermal conductivity overestimates the experimental heat transport in the pedestal because the mean free paths of both species are longer than estimates of the parallel thermal correlation lengths, and collisionless transport models are probably required for accurate description. However, even the collisionless estimates for electron thermal transport are too large by one to two orders of magnitude. Thus, it is likely that another mechanism such as rotational screening of resonant perturbations limits the stochastic region and reduces transport inside of the pedestal.

PACS: 05.45.Gg, 52.55.Fa, 52.55.Rk, 52.65.-y 


\section{Introduction}

The strike point structure of a poloidally-diverted tokamak has been predicted to become manifestly non-axisymmetric and develop helically striped patterns [1-6] when perturbed by magnetic fields due to external coils, field errors, and internal modes. There is potential to optimize this "strike-point splitting" in order to spread heat flux during steady-state operation in next-generation diverted reactors. Such patterns have been observed experimentally on DIII-D [7-9] during the application of three-dimensional magnetic perturbations and during locked modes. Non-axisymmetric heat flux deposition patterns during edge localized modes (ELMs) have also been observed on ASDEXUpgrade [10-11] and during locked modes leading to disruption on MAST [12]. In addition, resonant magnetic perturbations (RMPs) have been shown to suppress ELMs in H-mode plasma discharges in both DIII-D [13-16] and JET [17]. With edge-resonant $n=3$ perturbations, a low-density regime of steady-state ELM-free operation can be achieved in DIII-D [15-16], so that ELM-quiescent divertor operation is actually realized. This is important because at ITER values of pedestal collisionality, ELMs are predicted to release up to $20 \%$ of the pedestal stored energy on timescales fast enough to significantly erode the divertor target plates [18-19] and, thus, divertor lifetime.

Simulation of heat transport for DIII-D H-mode plasma discharge 122342 during the RMP ELM-suppressed phase is performed using the E3D Braginskii fluid transport code [20-21]. The E3D code, described in Section 2.2, performed the first calculations of nonaxisymmetric heat flux deposition [20] for the circular limited TEXTOR tokamak's Dynamic Ergodic Divertor [22]. In this work, we use the E3D code to examine the heat flux patterns achieved in a poloidally diverted system and to address the question of 
whether stochastic transport can be responsible for the reduction in pedestal pressure gradient that led to the stabilization of Type-I peeling-ballooning modes as proposed in Ref. [16].

DIII-D's strongly shaped magnetic fields are reconstructed using the TRIP3D code [23] which superimposes the fields from a given set of external coils upon the axisymmetric Grad-Shafranov EFIT [24] equilibrium reconstructed from experimental magnetic probes, flux loops, and motional Stark effect measurements. In order to reconstruct plasma profiles, kinetic EFIT [25] profile reconstructions utilize information from Thomson scattering and charge exchange recombination spectroscopy, as well as calculations of the fast-ion pressure and bootstrap current. In addition to external perturbations from the internal MHD control coil (I-coil) and the field-error correction coil (C-coil), TRIP3D can also use a detailed model of intrinsic field errors constructed by the DIII-D field-error analysis team [26]. The I-coil consists of 6 upper and 6 lower sets of coils and is shown in Refs. [13-16]. All discharges in this study correspond to the up-down symmetric $n=3$ "even-parity" configuration described in Refs. [15] and [16] that has six times larger resonant perturbation, $\delta B_{m n} / B$, than that of the up-down asymmetric "odd-parity" configuration described in Refs. [8] and [13].

A Poincaré plot of the $\phi=0$ surface of section relative to the normalized poloidal magnetic flux surfaces, $\psi$, produced by TRIP3D for shot 123301 at $2170 \mathrm{~ms}$ is shown in figure. 1(a). Field lines that escape to the divertor target have been traced for a maximum of 200 toroidal transits and are colored from yellow to black (color online) by the number of toroidal transits before escape in the forward direction (from 200 to 1). The Poincaré plot implies that many of the field lines in the outer $25 \%$ of the plasma (in $\psi$ ) can escape 
within $\sim 1-2 \mathrm{~km}$. Remarkably, the measured plasma temperatures remain high enough $\sim 1-2 \mathrm{keV}$, that these connection lengths are of the same order of magnitude as the electron mean free path in this region [27]. In addition, we will find that the mean free paths of both species are longer than the thermal correlation lengths that are expected in the stochastic field. This implies that collisionless processes are important for determining parallel transport along field lines.

Previous E3D simulations [9] have demonstrated greatly enhanced stochastic transport when all RMP sources are used. Because the C-coil and field errors introduce significant $n=1$ perturbation components that penetrate deeply into the plasma, the stochastic region is relatively broad and a significant fraction of field lines can escape from the location of the unperturbed $q=2$ flux surface [27]. However, an important caveat in the TRIP3D analysis is that the simple superposition of the equilibrium and external fields allows complete reconnection of magnetic flux. Unless the perturbation induces large enough Lorentz braking forces at the resonant surfaces to overcome the force of viscous friction in the bulk plasma, plasma rotation will tend to shield the resonant magnetic perturbations [28-32]. This screening well known to occur in the core, where the rotation is large and the resistivity is small. Additionally, Heyn, et al., [32] found that the electron diamagnetic flow at the edge of the H-mode plasma is large enough to limit the penetration of the resonant fields at the edge. For simplicity, in this work we will not attempt to provide an accurate description of the plasma response fields. Instead, we will present results that assume that the TRIP3D analysis provides a first approximation of the magnetic field sufficiently close to the separatrix. In order to limit the perturbation to the edge, in the following we study the effect of the I-coil perturbation 
alone. Although the fields in this case are relatively weakly stochastic, they have significant impact on the energetic balance in the simulation and the pattern of heat flux delivered to the divertor target.

In Section 2 we explore estimates for the stochastic enhancement of thermal diffusion and describe the computational technique required to accurately compute the parallel thermal transport in the fluid regime. We then describe the experimentally obtained transport results and compare them to results of E3D simulation. In Section 3 we describe the way in which heat flux is guided to the divertor target within the simulation and compare the TRIP3D and E3D calculations of strike point splitting to experimental measurements. Finally, we conclude with a discussion of the relevance of our results to experimentally determined transport and summarize our conclusions. 


\section{Stochastic Heat Transport}

\subsection{Quasi-linear estimates}

The dynamics of magnetic field lines can be estimated from the spectrum of the resonant magnetic field normal to a magnetic surface. The axisymmetric equilibrium field has good flux surfaces, and thus, has the action-angle form $\mathbf{B}_{0}=\nabla \Psi_{P} \times \nabla(q \theta-\phi) / 2 \pi$, where $\phi$ is the usual cylindrical toroidal angle and $\Psi_{P}=\oint \mathbf{B} \cdot d \mathbf{a}_{P}$ is the magnetic flux (in $\mathrm{V} \mathrm{s}$ ) contained by a surface of constant poloidal angle. The poloidal angle is chosen to be a straight field line coordinate so that $\theta=\left(\phi-\phi_{0}\right) / q$ and the safety factor $q$ is a function of $\Psi_{P}$ alone. If we define the toroidal flux contained by a surface of constant toroidal angle $\Psi_{T}=\oint \mathbf{B} \cdot d \mathbf{a}_{T}$, then the rotational transform $\iota=1 / q=d \theta / d \phi=d \Psi_{T} / d \Psi_{P}$ measures the change in poloidal angle for each toroidal revolution. In the following, we normalize the poloidal flux $\psi=\left(\Psi_{P}-\Psi_{\text {axis }}\right) / \Psi_{0}$ to the total poloidal flux contained within the plasma $\Psi_{0}=\Psi_{\text {sep }}-\Psi_{\text {axis }}$. The Fourier series coefficients of the surface averaged magnetic field perturbation normal to the unperturbed flux surfaces are defined via

$$
\delta B_{m n} A=2 \oint \sin \left(m \theta-n \phi+\chi_{m n}\right) \delta \mathbf{B} \cdot d^{2} \mathbf{a},
$$

where $A$ is the area of each flux surface and the phases of the coefficients are $\chi_{m n}$. With this surface averaged definition, the perturbation to the normal field can be produced either by the perturbed poloidal flux function

$$
\tilde{\Psi}_{P}=\sum_{m n} \frac{\delta B_{m n} A}{2 \pi m} \cos \left(m \theta-n \phi+\chi_{m n}\right)
$$

for $m \neq 0$, or by the perturbed toroidal flux function 


$$
\tilde{\Psi}_{T}=\sum_{m n} \frac{\delta B_{m n} A}{2 \pi n} \cos \left(m \theta-n \phi+\chi_{m n}\right)
$$

for $n \neq 0$. The exact geometric dependence of the majority of the formulae below can be captured by normalizing the magnetic perturbation to the total poloidal flux, $b_{m n}=\delta B_{m n} A / 2 \pi \Psi_{0}$. Figure 1(b) displays the spectrum of the $n=3$ component of the Icoil perturbation at $3 \mathrm{kA}-\mathrm{t}$ as modeled by TRIP3D for plasma discharge 122342 at $4650 \mathrm{~ms}$. Note that the spectrum has a ridge that follows the resonant value $m=-3 q$. Near the separatrix, the peak values are on the order of $1.8 \times 10^{-3}$.

The spectrum determines the island width, $\delta \psi=4 \sqrt{b_{m n} / q^{\prime} n}$, where $q^{\prime}=d q / d \psi$, and since resonances are separated as $\Delta \psi=1 / q^{\prime} n$, one can also estimate the Chirikov island overlap parameter [33], $\sigma=\delta \psi / \Delta \psi=4 \sqrt{q^{\prime} n b_{m n}}$. As the separatrix is approached, the reconstructed profile is expected [3-4] to display logarithmic behavior of the form $q \rightarrow q * \ell n(h * / h)$, where we define $h=1-\psi$, and $q *, h *$ are constants to be determined. The large shear $q^{\prime} \rightarrow q^{*} / h$ forces island sizes to decrease as $h^{1 / 2}$ as the separatrix is approached, but since the width between resonances decreases in proportion to the shear, the island overlap still increases as $h^{-1 / 2}$. Thus, transition to stochasticity generically occurs sufficiently close to the separatrix at $h_{s t}=16 q_{*} n b_{m n}$. A fit to the reconstructed $q$ profile produces $q_{*}=0.56, h_{*}=24$, and therefore $h_{s t} \sim 5 \%$. Figure 2(a) shows that, for the I-coil perturbation alone, the overlap parameter does in fact exceed unity very close to this location. Since the island half-width at this location is $3 \%$, this additional width must be included within the overlap region as shown in the figure. Note however, that due to the effect of 2nd order islands, overlap generically occurs sooner than this; Chirikov [33] 
found the more stringent criterion $\sigma>2 / 3$. This estimate would yield an outer stochastic layer of roughly $15 \%$ width in poloidal flux.

Knowledge of the perturbation spectrum allows one to estimate the rate at which magnetic field lines wander across flux surfaces. For sufficiently ergodic motion, the field lines move across the unperturbed flux surfaces in a diffusive fashion so that $\left\langle\Delta x^{2}\right\rangle \sim 2 d_{f l} \ell$ along the length of a field line. The quasi-linear estimate [34] for the diffusion of poloidal flux in poloidal angle defined via $\left\langle\Delta \psi^{2}\right\rangle=2 D_{\psi} \Delta \theta$,

$$
D_{\psi}=\frac{\pi}{2} \sum_{n} b_{m n}^{2} \delta_{m-q n}
$$

grows quadratically with RMP amplitude. Here, $\delta_{m-q n}$ is shorthand for the Kronecker delta function $\delta_{m-q n, 0}$. This, in turn, allows one to estimate the diffusion of field lines in real space

$$
d_{f l}=\frac{B_{T}}{q R B} \frac{D_{\psi}}{|\nabla \psi|^{2}} \approx \frac{\pi q R}{2} \sum_{n}\left(\frac{\delta B_{m n}}{B_{T}}\right)^{2} \delta_{m-q n},
$$

where $B_{T}$ is the toroidal field, $B$ is the total field strength and $R$ is the major radius at the magnetic axis. Figure 2(b) shows that the characteristic diffusion coefficient is on the order of $1 \mathrm{~mm}^{2} / \mathrm{m}$. In the collisionless regime, heat will diffuse at the thermal speed of the carrier particles [35-36], so that one can estimate the thermal diffusion coefficient to be $D_{s t}=d_{f l} V_{T}$ where the thermal velocity $V_{T}=\sqrt{T / M}$ is defined in terms of the temperature $T$ and mass $M$ of the particle species under consideration. 
In the collisional regime where the mean free path is shorter than the parallel thermal correlation length scale, $\lambda \ll L_{T}$, Rechester and Rosenbluth [35] estimated that the thermal transport coefficient would be reduced by a factor of $\lambda / L_{T}$, so that $D_{R R}=D_{S t} \lambda / L_{T}=D_{\|} d_{f l} / L_{T}$. In equilibrium, the ratio of perpendicular $\left(\rho_{T}\right)$ to parallel ( $L_{T}$ ) correlation length scales must satisfy $\rho_{T} / L_{T}=\sqrt{D_{\perp} / D_{\|}}$in order to balance the diffusive time scales along and across magnetic field lines. At the same time, motion in the stochastic field causes these small perpendicular scales to expand (along the unstable invariant manifolds described below) until they extend over a poloidal island length $r / m=\rho_{T} \exp \left(L_{T} / L_{K}\right)$. Thus, the parallel thermal correlation length scale should be determined by the largest Liapunov exponent, or Kolmogorov length, up to a correction factor that only depends logarithmically on the diffusivity ratio,

$$
L_{R R}=L_{K} \ln \left(\frac{r / m}{L_{R R}} \sqrt{\frac{D_{\|}}{D_{\perp}}}\right)
$$

The Kolmogorov length can be estimated via [37-38]

$$
\frac{L_{K}}{\pi q R}=\frac{2 \Gamma\left(\frac{1}{3}\right)}{\pi \Gamma\left(\frac{2}{3}\right)}\left(3 D_{K}\right)^{-1 / 3} \approx 1.10\left(D_{K} / 2\right)^{-1 / 3},
$$

where $D_{K}$ represents the diffusion of neighboring trajectories. The quasi-linear estimate for $D_{K}$ is given by a higher spectral weight of the perturbation spectrum,

$$
D_{K}=\frac{\pi}{2} \sum_{n}\left(q^{\prime} n b_{m n}\right)^{2} \delta_{m-q n}
$$


Since the Kolmogorov length is expected to scale inversely with perturbation strength, $L_{K} \sim b^{-2 / 3}$, the collisional diffusivity scaling, $D_{R R} \sim d_{f l} / L_{K} \sim b^{8 / 3}$, is even more rapid than the quadratic scaling in the collisionless case.

These estimates are clearly only relevant if a field line is long enough to sample the stochastic region, so that $\lambda \ll L_{K} \ll L_{\mathcal{C}}$. If the connection length to the wall, $L_{\mathcal{C}}$, becomes shorter than the Kolmogorov length, then the field lines exit the plasma ballistically, not diffusively, and this leads to greatly enhanced parallel transport [38]. Far enough from the separatrix, the poloidal connection length, $L_{\mathcal{C}} \sim \pi q R$, yields a reasonable estimate; however, in the stochastic case the connection length develops fractal structure [3-4,20]. Thus, we define the parallel collisionality by the ratio of the parallel thermal correlation length to the mean free path:

$$
\ell *=L_{T} / \lambda \sim \min \left(L_{R R}, L_{c}\right) / \lambda,
$$

where the asymptotic scalings hold in the appropriate regime. This definition is clearly different from the definition of perpendicular collisionality [39] that compares the collision frequency to an effective neoclassical bounce frequency.

\subsection{Computational method}

The E3D code [19-20] uses Monte-Carlo fluid elements to efficiently integrate the highly anisotropic advection-diffusion Braginskii fluid equations. Because the code has not yet implemented a model for neutral refueling, a key component of $\mathrm{H}$-mode pedestal density physics, we focus on thermal transport alone, and keep the density fixed throughout the simulation. In this case, the solutions to the energy conservation equations

$$
\partial_{t} \frac{3}{2} n_{e} T_{e}=\nabla \cdot n_{e} \chi_{\| e} \hat{\mathrm{b}} \hat{\mathrm{b}} \cdot \nabla T_{e}+\nabla_{\perp} \cdot n_{e} \chi_{\perp} \nabla_{\perp} T_{e}+Q_{e i}
$$




$$
\partial_{t} \frac{3}{2} n_{i} T_{i}=\nabla \cdot n_{i} \chi_{\| i} \hat{\mathrm{b}} \hat{\mathrm{b}} \cdot \nabla T_{i}+\nabla_{\perp} \cdot n_{i} \chi_{\perp} \nabla_{\perp} T_{i}-Q_{e i}
$$

are essentially entirely determined by the boundary conditions and the geometry of the magnetic field. The parallel diffusivities are defined by their Braginskii values [40]

$$
\chi_{\| e}=3.2 \tau_{e i} V_{T_{e}}^{2} \quad \chi_{\| i}=3.9 \tau_{i i} V_{T_{i}}^{2}
$$

where $\tau_{e i}\left(\tau_{i i}\right)$ represent the Coulomb collision time between ions and electrons (ions). The collisional heating of electrons by ions is simply given by

$$
Q_{\mathrm{ei}}=3 n\left(T_{\mathrm{i}}-T_{\mathrm{e}}\right) m_{\mathrm{e}} / m_{\mathrm{i}} \tau_{\mathrm{ei}}
$$

since relative flows are neglected. The nonlinear boundary conditions applied at the walls are the so-called Bohm sheath boundary conditions,

$$
q_{\alpha}=\delta_{\alpha} T_{\alpha} \Gamma_{\alpha} \quad \Gamma_{\alpha}=n_{\alpha} C_{\mathrm{s}} \cos \beta_{w},
$$

for each species $\alpha$, that require outflow of particles and energy at the ion sound speed, $C_{s}$. The boundary conditions depend on the angle between the field line and the wall $\beta_{w}$, and the flux coefficients $\delta_{e}$ and $\delta_{i}$ are determined from kinetic modeling studies in [41]. The plasma is assumed to be quasi-neutral so that $n_{e}=Z n_{i}$ with constant charge state $Z=1$. The perpendicular diffusivities are assumed to be anomalous in origin and can be chosen to match experimental conditions. Experimentally inferred values for DIII-D Hmode plasma profiles typically range from $0.1-10.0 \mathrm{~m}^{2} / \mathrm{s}$ and vary significantly over the spatial extent of the edge plasma.

The Monte-Carlo elements are not particles, but heat packets which satisfy the equations of motion

$$
\Delta \vec{x}=\sqrt{2 \Delta t \vec{D}} \cdot \vec{\xi}+\vec{V} \Delta t
$$


where $\vec{\xi}$ is a vector of random numbers with unit standard deviation. In the limit of small time steps, the distribution function $f(x)=\sum_{i} w_{i} \delta\left(x-x_{i}\right)$ for the packets of heat, particles, etc. satisfies the ensemble-averaged $\langle f\rangle$ equation of motion

$$
\frac{d\langle f\rangle}{d t}+\vec{\nabla} \cdot(\vec{V}-\vec{\nabla} \cdot \vec{D})\langle f\rangle=S-v\langle f\rangle
$$

The fluid equations of motion are reproduced with the choice $\vec{V}_{\text {packet }}=\vec{V}_{\text {fluid }}+\vec{\nabla} \cdot \vec{D}$ and sources represent the probability to inject or remove heat packets.

Because the thermal diffusivity is so anisotropic, $\chi_{\|} / \chi_{\perp} \sim 10^{8}-10^{11}$, tracking large parallel jumps of heat packets represents one of the major computational costs of the algorithm. E3D efficiently handles this cost by pre-computing field line following grids that trivialize the integration of the equations of motion. E3D uses the technique of "multiple local magnetic coordinate systems" to generate a series of field line following meshes that cover the entire domain. The RMP fields of interest are much smaller than the main toroidal field; by cutting the domain into enough toroidal sections, one can find a locally straight magnetic field line coordinate system in each subdomain. To make the method global, E3D generates a 3rd order spline mapping that interpolates between the faces of the subdomains. Thus, the large number of parallel numerical integration steps can be exchanged for a greatly reduced number of map evaluations. We find that six toroidal subdomains reproduce the field motion to sufficiently high accuracy. The mapping between subdomains typically has 80 radial x 360 poloidal grid points, while the plasma parameters within each subdomain are stored on a 32 or 64 point radial mesh $\mathrm{x}$ 360 poloidal x 16 toroidal mesh. A flexible definition of poloidal angle is used to focus 
enough resolution over the divertor region and on the divertor target to resolve the magnetic footprint.

\subsection{Experiment}

During DIII-D discharge 122342, the I-coils are energized to $3 \mathrm{kA}-\mathrm{t}$ in the $n=3$ even parity configuration at $2000 \mathrm{~ms}$, just after H-mode transition. The standard field-error correction algorithm was applied to the C-coil (maximum current of $12 \mathrm{kA}-\mathrm{t}$ ) throughout the discharge. When the edge-safety factor reaches $q_{95} \sim 3.7$, particle transport is strongly affected, and the line-averaged density begins to decrease. Just after the coils are energized, the ELM frequency increases while the ELM size decreases until the density drops low enough to reach the point of marginal ELM stability. The ELM frequency then decreases until ELMs are completely suppressed at $2900 \mathrm{~ms}$, when the target edge safety factor, $q_{95}=3.5$, is achieved and the pedestal density is reduced to $n_{e, p e d}=2 \times 10^{13} \mathrm{~cm}^{-3}$. Time-traces of similarly shaped discharges can be found in Refs. $[15,16]$.

Figure 3(a) displays a set of fits to electron density, temperature, and ion density derived from Thomson scattering and charge exchange recombination data for discharge 122342 averaged over the ELM suppressed interval 4400-4900 ms. At $4650 \mathrm{~ms}$, the total input power is $P_{N B I}+P_{O h}=5.1 \mathrm{MW}$ and the stored energy is essentially constant. The core-radiated power is $P_{\text {rad,core }}=0.5 \mathrm{MW}$, while the divertor radiates $P_{\text {rad, } d i v}=0.9 \mathrm{MW}$.

On axis, $n_{e}(0)=4 \times 10^{13} \mathrm{~cm}^{-3}$, and the low-density operation appears to increase the deposition of neutral beam energy into the ion channel: the ions reach $9 \mathrm{keV}$ on axis while the electrons reach $3.5 \mathrm{keV}$. At the inside of the pedestal, $\psi=0.85$, the ion 
temperature is $2.5 \mathrm{keV}$, while the electron temperature is $1.3 \mathrm{keV}$. Transport modeling using the comprehensive CORSICA code suite [42] determined that pedestal ions received close to twice as much power as pedestal electrons.

Figure 3(b-d) represent estimates of the parallel thermal diffusivity, mean free path, and correlation lengths based on these experimental profiles, using the expressions developed in Section 2.1. They will be discussed in detail in Section 2.5.

\subsection{Simulation}

We perform two numerical experiments that are intended to roughly match experimental conditions during the axisymmetric and RMP ELM-suppressed state. The protocol is to initialize E3D with axisymmetric profiles that are close to satisfying the correct boundary conditions and evolve until steady state. The density is not evolved, instead it is chosen to be a flux function that fits experimental Thomson scattering data, but becomes constant beyond the separatrix [figure 4(a,b)]. Because E3D requires a good flux surface as an inner boundary condition, the inner surface is placed at $\psi=0.86$ in the unperturbed case and $\psi=0.82$ in the perturbed case. The simulation domain extends outward to include a realistic vacuum vessel where the sheath boundary conditions [equation (13)] must be satisfied. At the point of closest approach on the outer midplane, the vaccum vessel wall lies at a normalized flux of $\psi=1.20$. Since the stochastic field penetrates deeply into the plasma, a simple 1 or 2 level diffusivity model is not accurate enough to reproduce the experimental temperature profiles across the entire plasma domain. According to the CORSICA analysis, the diffusivity of total pressure from the observed profiles, $D_{\perp} \sim 1 \mathrm{~m}^{2} / \mathrm{s}$ at the top of the pedestal and $D_{\perp} \sim 0.2 \mathrm{~m}^{2} / \mathrm{s}$ in the region of steep gradients. However, the steep gradient region is too narrow to accurately 
resolve in the simulation and qualitative results for the footprint structure are expected to be somewhat insensitive to the diffusivity profile; thus, we choose $D_{\perp}=1 \mathrm{~m}^{2} / \mathrm{s}$ to be constant throughout the domain. In the simulation, a constant heat flux of $5 \mathrm{MW}$ is applied to the inner boundary, and consistent with the power fraction determined by CORSICA, 3.3 MW of the power is injected into the ions, while 1.7 MW is injected into the electrons.

Simulation results are shown in figures 4 and 5. The unperturbed cases are close to axisymmetric, while the perturbed cases display three-dimensional structure (figure 5) that is especially obvious near the X-point where there is large flux expansion. In the next section it will be shown that this structure is primarily determined by the threedimensional magnetic field line topology induced by the RMP. Figure 4(b) displays the simulation density and temperature profiles across the outer midplane. In the unperturbed case (0 kA-t), the profiles are roughly linear due to the choice of constant diffusivity and the temperatures achieved on the inner boundary are $1.7 \mathrm{keV}$ for ions and $1 \mathrm{keV}$ for electrons. The profiles in the perturbed case (3 kA-t) are linear as well, but have suffered a large drop in thermal confinement: the temperatures achieved on the inner boundary are $0.9 \mathrm{keV}$ for ions and $0.4 \mathrm{keV}$ for electrons.

\subsection{Thermal Transport Comparison}

Considering the discussion of stochastic transport in the previous sections, it is surprising that the experimental thermal confinement remains high while particle confinement is reduced. In Refs [15] and [16] it was demonstrated that the temperatures of both species in the steep gradient region are actually higher than the pre-RMP ELMing phase. In these experiments, the RMP reduces particle confinement at roughly constant 
stored energy. This implies a trade-off between higher density during the pre-RMP phase for higher temperature during the RMP phase. However, there appears to be a large discrepancy between experiment and simulation in the RMP-induced change to the stored energy. The simulation results clearly show an enhancement to thermal conductivity in agreement with stochastic transport expectations.

To understand the source of the discrepancy qualitatively, let us examine the consequences of the quasi-linear transport estimates described in Section 2.1. Due to the relatively light mass of electrons, we expect electron thermal conduction to be 60 times larger than that of deuterium ions at fixed temperature. The Braginskii parallel thermal diffusivities in figure $3(\mathrm{~b})$, defined via $D_{\|}=2 \chi_{\|} / 3$, range from $10^{11} \mathrm{~m}^{2} / \mathrm{s}$ for electrons to $10^{9} \mathrm{~m}^{2} / \mathrm{s}$ for ions over much of the observed plasma pedestal. The mean free paths for thermal conduction, defined via $\lambda=2 \chi_{\|} / 3 V_{T}$, for the experimental plasma are on the order of $1-4 \mathrm{~km}$ at the top of the pedestal as shown in figure 3(c). Due to the logarithmic correction factor, the thermal correlation lengths are close to 200-400 m, about 2-4 times larger than the Kolmogorov length [figure 3(d)]. Thus, even if we neglect the transition to short connection length at the plasma edge and use the approximation $\ell *=L_{R R} / \lambda$, figure 6(a) shows that the $\mathrm{H}$-mode plasma is quite collisionless since $\ell *_{e} \sim 0.2$ and $\ell_{* i} \sim 0.03$. In fact, the ions are less collisional than the electrons due to their higher temperature.

Quasi-linear estimates for diffusive transport over the entire pedestal are shown in figure 6(b,c). Note that, since the collisional result $D_{R R}=D_{s t} / \ell *$ should only be used when $\ell_{*}>1$, the appropriate estimate is always the minimum of the two possible values. In figure $6(b, c)$, the physically correct estimate is indicated by open symbols. For the 
experimental H-mode profiles, the estimated collisionless electron thermal diffusivity reaches a maximum of $\sim 20 \mathrm{~m}^{2} / \mathrm{s}$ in the steep gradient region near $\psi=0.97$. Recall that the experimental result is closer to $0.2 \mathrm{~m}^{2} / \mathrm{s}$, two orders of magnitude smaller, and both the observed electron and ion temperatures actually increase during the RMP [15-16]. The collisionless ion thermal diffusivity, $D_{s t}=0.3-0.4 \mathrm{~m}^{2} / \mathrm{s}$ over the range $\psi=0.95-1.00$, does have the correct order of magnitude to explain the increase in effective particle transport. However, such a change in thermal conductivity is large enough to compete with neoclassical transport and should have observable consequences in the steep gradient region.

In contrast, for the simulated profile set, the temperatures of both species reach much lower values, and for the perturbed cases, this reduces the parallel diffusivities [figure 3(b)] and mean free paths [figure 3(c)] by one to two orders of magnitude. In the simulation, the electrons are cold enough to actually become collisional at these low densities and the ions are close to marginal, $\ell_{*_{i}} \sim 1$, in the outer $5 \%$ of the plasma [figure 6(a)]. Thus, we can conclude that, under the simulated conditions, the fluid plasma model is valid for electrons and marginal for ions. Figure $6(\mathrm{~b}, \mathrm{c})$ show that the simulated stochastic enhancement in thermal diffusivity is roughly $D_{R R e} \sim 2 \mathrm{~m}^{2} / \mathrm{s}$ for electrons and $D_{R R i} \sim 0.5 \mathrm{~m}^{2} / \mathrm{s}$ for ions over much of the simulated pedestal. These order of magnitude Rechester-Rosenbluth estimates agree with the enhancement of diffusivity implied from the drop in the temperatures between simulations: $\Delta D_{e} \sim$ $1.5 \mathrm{~m}^{2} / \mathrm{s}$ for electrons and $\Delta D_{i} \sim 0.7 \mathrm{~m}^{2} / \mathrm{s}$ for ions. 


\section{Strike Point Splitting}

\subsection{The homoclinic tangle}

In order to understand the way in which heat is transported across the separatrix, it is imperative to study the behavior of field lines in the vicinity of the separatrix and the $\mathrm{X}$-point in particular. The X-point is constructed from four families of field lines: as we trace field lines in the forward toroidal direction, there are two entering families and two exiting families. In the axisymmetric case shown in figure 7(a), the entering and exiting field lines coincide and form the separatrix from a single self-intersecting, or homoclinic, orbit. Under perturbation, the X-point trajectory will deform, but it will survive. However, self-intersecting orbits are structurally unstable [43-45], and this topology will be destroyed by an arbitrarily small perturbation, as in figure 7(b). The four branches of the X-point can still be determined by locating the eigenvectors of the toroidal mapping of field lines near the fixed point and tracing the motion of field lines that enter/exit the $\mathrm{X}$-point along those eigenvectors. However, "time reversal" symmetry in toroidal angle is fundamentally broken along the separatrix. Field lines that enter the X-point in the forward direction define the so-called forward stable (backward unstable) invariant manifold, while the field lines that enter the X-point in the reverse direction define the forward unstable (backward stable) invariant manifold, and these two manifolds no longer coincide [43-45].

No field line can ever cross an invariant manifold, because the manifold is explicitly constructed from field lines, and thus, must be invariant under parallel translations, $\vec{B} \cdot \vec{\nabla} \psi=0$. However, in a chaotic field, these surfaces represent "non-isolating" 
constants of the motion, and they do not restrict field lines from wandering ergodically throughout the stochastic area of phase space. If we initialize points inside of the original separatrix and follow the field lines as they travel in the forward toroidal direction until they first strike the divertor target, figure 7(c) shows that they fill a large stochastic region, but the motion is clearly still bounded by a well-defined outer surface. Similarly, figure 7(d) shows that when traced in the opposite direction, a different outer bounding surface is developed. Neither manifold can intersect itself, but they generically intersect one another. If they intersect once, they must intersect an infinite number of times in either direction because the point of intersection can be mapped forward/backward along the stable/unstable manifold an infinite number of times, cf. figure 7(b). The lobes that lie between the stable and unstable manifolds transport flux along the boundary, and because the mapping is area preserving, both the forward and backward mapping of each lobe contains an equivalent amount of flux. The points of intersection of the manifolds must accumulate near the X-point, where the poloidal field vanishes, and, in order to preserve area, the lobes must then lengthen in order to counteract the decrease in width. As the lobes lengthen, their tips wander along an ever-increasingly complex self-avoiding path, eventually mixing with other lobes. This mixing is ballistic at first since the lobes are simply transported along the direction of the local pitch of the field lines on the separatrix, but after a close-encounter with the X-point, a field line will be scattered in an effectively random fashion [46-47].

Although the separatrix topology is "broken" by the perturbation, the invariant manifolds in the vicinity of the original separatrix still completely determine the fate of field lines. Melnikov [43] proved that small deformations of such manifolds can be 
treated linearly. To find the distance between the stable $\psi_{-}$or unstable $\psi_{+}$manifold and the original separatrix $\psi_{0}$, simply integrate along a field line from a point infinitesimally close to the X-point,

$$
\psi_{ \pm}(\phi, q \theta)=\psi_{0}+\int_{\mp \pi}^{\theta} \frac{\vec{B} \cdot \vec{\nabla} \psi}{\vec{B} \cdot \vec{\nabla} \theta} d \theta
$$

The difference in flux between the two manifolds, the Melnikov function,

$$
\delta h\left(\phi_{0}\right)=\psi_{+}-\psi_{-}=\int_{-\pi}^{\pi} \frac{\vec{B} \cdot \vec{\nabla} \psi}{\vec{B} \cdot \vec{\nabla} \theta} d \theta=\underset{n}{2 \pi \sum_{m n}}\left(\psi_{0}\right) \delta_{m-q n} \sin \left(\chi_{m n}-n \phi_{0}\right)
$$

depends upon the constant of motion $\phi_{0}=\phi-q \theta$ alone. Note that although $\theta$ becomes singular as the separatrix is approached, the product $q \theta$ is simply the toroidal angle traversed, and thus, remains well behaved. The zero crossings give the primary points of intersection between the manifolds, while the integral of this function can be used to measure the area between the lobes. The distance in the direction normal to the original separatrix, increases as the X-point is approached $\hat{n} \cdot \delta \vec{x}=\delta h /\left|\nabla \psi_{0}\right|$, but in order to preserve the area between the lobes, the distance between crossings decreases proportionately. Note that the radial excursions are linear in the perturbation field.

The accessible stochastic region is asymmetric with respect to the unperturbed separatrix: it is thicker on the interior than the exterior. The half width of the layer described by the Melnikov funciton, $\delta h \sim 2 \pi b_{m=q n} \sim 1 \%$, is only $4 \mathrm{~mm}$ on the outer midplane, but due to the expansion of the unperturbed flux near the X-point, it creates a $2 \mathrm{~cm}$ footprint on the divertor floor. It is much thinner than the stochastic layer predicted by island overlap $h_{s t} \sim 5 \%$, and the ratio is 


$$
\delta h / h_{s t}=\pi / 8 q * n,
$$

close to $1 / 5$. Thus, as particles move along field lines, they leave the relatively wide stochastic layer, mix into the relatively thin homoclinic tangle, and eventually strike the divertor target. Upon reflection/re-injection through recycling processes they travel in the opposite direction, sampling the entire region between the two upper invariant manifolds. Just outside of the homoclinic tangle, particles cannot mix with the interior, except through perpendicular transport mechanisms such as collisions, cross-field convection, or neutral transport. Thus, the outer envelope of the tangle provides a precise definition of the inner boundary of the laminar scrape off layer (SOL). In the SOL, field lines do not close on themselves, and thus, due to the rectification theorem [44-45], a straight field line coordinate system can be always be found locally. One expects exponential decay in this region; e.g. for the collisional fluid regime, the SOL decay length should satisfy $\rho_{S O L} / L_{C} \sim \sqrt{D_{\perp} / D_{\|}}$. By similar reasoning, the original private flux region below the $\mathrm{X}$ point is still magnetically well isolated, and will still have a rapid decay length due to the small connection length in this region. To verify this picture of field-aligned thermal transport, in figure 8 we compare the electron temperature calculated by E3D with the invariant manifolds that enter/exit the X-point for the mapping that defines the E3D coordinate system. Strong correlation is found between the invariant manifold structure and the contours of constant temperature.

Note that if there is not enough input power into the stochastic layer, it could reach temperatures below ionization threshold, and retain only an exponentially small fraction of plasma. In this case, the plasma edge will be defined by the last dominant tangle of island chains above ionization threshold. Such behavior is commonly predicted and 
observed in limiter configurations such as TEXTOR $[22,46]$; however, in the diverted system, the homoclinic tangle still defines an outer boundary that geometrically confines the invariant manifolds of the island chain within. In this case, the heteroclinic intersection [44-45] of the invariant manifolds of the island chain and the remnant of the poloidal separatrix defines the chaotic web in which trajectories wander out to the divertor.

\subsection{Magnetic footprints}

During $n=3$ even parity I-coil operation, both the inner and outer strike point (ISP/OSP) particle flux has been observed to split into multiple striations. For example, images of $D_{\alpha}$-filtered recycling emission shown in figure 9(a) for shot 123301 clearly display three striations along the inner strike point, and this is also commonly observed in both CII and CIII filtered light. The detailed structure of the OSP has also been measured by slowly sweeping the OSP over a newly upgraded high-resolution Langmuir probe array (LPA) at the $\phi=180$ deg toroidal location. Multiple peaks have been observed in the radial profiles of floating potential [47]. The distribution of field lines that are initialized on unperturbed flux surfaces inside the unperturbed separatrix as they first strike the divertor target is shown in figure 9(b) as calculated for shot 123301 including all RMP sources: I-coil, C-coil, and field-errors. In good agreement with observations, TRIP3D predicts that there should be three striations along the inner strike point within the view of the tangential X-point TV, 120-240 deg. The IR camera view at 60 deg only observed a single striation which might be explained by the fact that the $n=1 \mathrm{C}$-coil and field error perturbations introduce significant squeezing of the magnetic footprint at this location [figure 9(b)]. However, further measurements at other toroidal locations have yet 
to observe significant striations in heat flux, and this would mean that the observed striations in particle flux are thermally isolated from the interior. More data analysis is needed to clarify the present experimental situation, but this conclusion would be consistent with the hypotheses of significant screening of resonant perturbations and/or parallel thermal transport inside the separatrix.

The simulated thermal footprints for shot 122342 with $5 \mathrm{MW}$ of input power and $3 \mathrm{kA}-\mathrm{t}$ of I-coil perturbation current (no C-coil or field errors) are shown in figure 10(a,b). The $n=3$ symmetry of the I-coil perturbations is apparent in the magnetic footprint structure. Because of the neglect of particle conservation, neutrals, and radiation, the computed heat fluxes are higher than expected for realistic conditions; the scales must be interpreted in light of the somewhat unphysical conditions of this numerical experiment. Although there was twice as much power injected into the ions 3.3 MW vs 1.7 MW, in both axisymmetric and perturbed cases, the heat flux to the divertor is more equally balanced between ions, $3 \mathrm{MW}$, and electrons, $2 \mathrm{MW}$, due to the collisional exchange of thermal energy. Since field lines that strike the inner divertor target are compressed to a smaller area, the heat flux is asymmetrically high even though only about $35 \%$ of the flux is channeled through the ISP both with and without RMP. Producing the correct inner/outer strike point asymmetry will eventually require drift physics and, perhaps, poloidal variation in the perpendicular transport coefficients. In fact, the ISP in DIII-D is typically detached, but during low-collisionality I-coil operation, the inner leg typically reattaches and delivers similar order of magnitude peak heat fluxes to the outer leg [48] as measured by infrared cameras. 
In the simulation, the peak heat flux of the toroidally averaged profiles is reduced by $17 \%$ for the ISP and $25 \%$ for the OSP. The toroidally averaged profiles of heat flux were fitted to the approximate exponential form, $Q \sim Q_{0} \exp (-\delta R / \rho)$, where $\delta R$ is the radial distance from the separatrix. While the decay lengths are unchanged within the private flux region, $\rho_{I S P} \sim 0.35 \mathrm{~cm}$ and $\rho_{O S P} \sim 0.57 \mathrm{~cm}$, on the scrape-off layer side, the decay length increases by $50 \%$ from 1.0 to $1.5 \mathrm{~cm}$ for the ISP and by $57 \%$ from $2.8 \mathrm{~cm}$ to $4.4 \mathrm{~cm}$ for the OSP. In fact, the toroidal average of the perturbed OSP profile appears to display an essentially linear, slower than exponential, decay.

Finally, a potential mechanism for enhanced convective transport is suggested by examining the heat flux sent directly to the entrance of the lower divertor cryopump. Simulated heat fluxes are delivered directly to the pump entrance at local levels of up to $10 \%$ of the peak value on the divertor. In addition, the mean position of the heat flux distribution, $\bar{R}=\int R \mathbf{q} \cdot \mathrm{d} \mathbf{a} / P$ with heat flux $\mathbf{q}$ and power $P$, over the OSP is moved $0.4 \mathrm{~cm}$ toward the pump entrance relative to the axisymmetric case. This average movement of plasma toward the pump may increase the probability that a neutral will reach the opening. This could increase pumping efficiency and, thus, decrease the effective recycling of particles back into the bulk plasma. 


\section{Conclusions}

In summary, we have calculated the non-axisymmetric distribution of heat flux to the DIII-D divertor under perturbation by the I-coil. The pattern is largely determined by the structure of the homoclinic tangle of invariant manifolds that guides field lines to the divertor target. The width of the magnetic footprint, the distribution of field lines escaping from the plasma region, can be thought of as the width of the effective separatrix. As the effective area for heat transport increases, the magnitude of the peak heat flux is reduced. The toroidal average of the perturbed profile decays slower than exponentially on the divertor target and indicates the increased efficiency with which heat flux is spread over the target. This also suggests that a linear decay of a "toroidally symmetric" strike point profile can potentially indicate toroidally rotating internal modes that produce an edge layer of stochastic field lines. The experimental observation of strike point splitting in particle flux appears to imply that the magnetic field in the SOL has developed significant non-axisymmetric structure. In addition, these observations coincide with changes to the pressure profile that are only observed during the application of edge-resonant perturbations.

Paradoxically, the observed change in the electron temperature profile does not agree with the weakly stochastic fluid simulations presented here. Theoretical analysis [35-38] and experimental results in L-mode and Ohmic plasmas [46,49-50] have consistently identified electron thermal conduction as the dominant transport mechanism. We have shown that $\mathrm{H}$-mode plasma parameters are collisionless even in the presence of weak stochasticity, and transport simulations at these densities will typically require kinetic treatment for accuracy when extended into regions with $\sim \mathrm{keV}$ temperatures. However, 
the collisionless quasi-linear estimates for parallel electron transport are too large by an order of magnitude at the top of the pedestal, and two orders of magnitude too large in the steep gradient region [16]. Thus, the non-axisymmetric structure developed by the magnetic field cannot thermally connect far into the pedestal. Current estimates of shielding by rotational effects [32] only allow penetration by a few $\%$ in poloidal flux.

In previous DIII-D RMP experiments, the largest factor in the reduction in pedestal pressure gradient was a drop in density due to increased particle transport [15-16]. Direct evidence for a factor of two reduction in the effective particle confinement time has been obtained by comparing pellet injection experiments in discharges with and without the RMP [51]. Ambipolar outflow aligned with the stochastic magnetic field should slow the net particle loss rate to that of the more massive ions [36,52-53]. The collisionless quasilinear estimate for the experimental ion diffusivity in Section $2.5, D_{\mathrm{st}}=0.3-0.4 \mathrm{~m}^{2} / \mathrm{s}$ over the range $\psi=0.95-1.00$, does have the correct order of magnitude to explain the observed reduction in particle confinement in the ion transport barrier region. However, this explanation requires a mechanism to reduce the conductive thermal transport of both species. Parallel electron conduction must be reduced by factor of 10 at the top of the pedestal and by a factor of 100 in the electron transport barrier region. The $1 \mathrm{D}$ transport model of Ref. [53] also found that agreement with experiment required an extreme factor of 10-30 reduction in the conductive thermal transport allowed in the collisionless kinetic regime. Many other explanations of particle transport have been proposed including: enhanced pumping efficiency (Section 3.2), enhanced neoclassical ion losses (Ref. [32]), static cross-field $\vec{E} \times \vec{B}$ convection cells (cf. the single fluid MHD model in Refs [5455]), and broadband dynamic turbulence (observed experimentally in Ref. [56]). 
Thus, it appears that the experimental results are inconsistent with a computational model of stochastic transport as well as quasi-linear estimates of thermal transport. It seems inevitable that another mechanism such as kinetic flux limits (cf. Ref. [53]) or the shielding of resonant perturbations by plasma rotation (cf. Ref. [32]) must play an important role in limiting stochastic transport. Because of the extreme sensitivity of the transport to the resonant perturbation strength, it is critical to understand the response of the plasma to the applied perturbations in order to predict the location and width of the resonant layer. 


\section{Acknowledgment}

This work was supported by the U.S. Department of Energy under DE-FC0204ER54698, DE-FG02-05ER54809, DE-FG02-04ER54758 and W-7405-ENG-48.

I. Joseph thanks the Max Planck Insitut für Plasmaphysik for providing supercomputing resources as well as accommodations in Greifswald, Germany. 


\section{References}

[1] Pomphrey N and Reiman A 1992 Phys. Fluids B 4938

[2] Punjabi A, Halima A, Boozer A 1997 Phys. Plasmas 4337

[3] Abdullaev S S and Finken K H 1998 Nucl. Fusion 38531

[4] Abdullaev S S, Finken K H, Jakubowski M and Lehnen M 2006 Nucl. Fusion 46 S113

[5] La Haye R J 1991 Nucl. Fusion 311550

[6] Roeder R K W, Rapoport B I, Evans T E 2003 Phys. Plasmas 103796

[7] Evans T E, Roeder R K W, Carter J A, et al 2005 J. Physics: Conf. Ser. 7174

[8] Evans T E, Joseph I, Moyer R A, et al 2007 J. Nucl. Mater. 363-365 570

[9] Joseph I, Moyer R A, Evans T E, et al 2007 J. Nucl. Mater. 363-365 591

[10] Eich T, Herrmann A, Neuhauser J, et al 2003 Phys. Rev. Lett. 91195003

[11] Eich T, Herrmann A, Neuhauser J, et al 2005 Plasma Phys. Control. Fusion 47815

[12] Delchambre E, Counsell G, Kirk A and Lott F 2007 J. Nucl. Mater. 363-365 1409

[13] Evans T E, Moyer R A, Thomas P R, et al 2004 Phys. Rev. Lett. 92235003

[14] Moyer R A, Evans T E, Osborne T H, et al 2005 Phys. Plasmas 12056119

[15] Burrell K H, Evans T E, Doyle E J, et al 2005 Plasma Phys. Control. Fusion 47 B37

[16] Evans T E, Moyer R A, Burrell K H, et al 2006 Nature Phys. 2419

[17] Liang Y, Koslowski H R, Thomas P R, et al 2007 Phys. Rev. Lett. 98265004 
[18] Loarte A, Saibene G, Sartori R, et al 2003 J. Nucl. Mater. 313-316 962

[19] Federici G, Andrew P, Barabaschi P, et al 2003 J. Nucl. Mater. 313-316 11

[20] Runov A, Kasilov S, Reiter D, et al 2001 Phys. Plasmas 8916

[21] Runov A, Kasilov S, McTaggart N, et al 2004 Nucl. Fusion 44 S74

[22] Finken K H and Wolf G H 1997 Fusion Eng. Des. 37337

[23] Evans T E, Moyer R A, and Monat P 2002 Phys. Plasmas 94957

[24] Lao L L, St John H E, Stambaugh R D, et al 1985 Nucl. Fusion 251611

[25] Lao L L, St John H E, Peng Q, et al 2005 Fusion Sci. and Tech. 48968

[26] Luxon J L, Schaffer M J, Jackson G L, et al 2003 Nucl. Fusion 431813

[27] Evans T E, Burrell K H, Fenstermacher M E, et al 2006 Phys. Plasmas 13056121

[28] Jensen T H, Leonard A W and Hyatt A W 1993 Phys. Fluids B 51239

[29] Boozer A H 1996 Phys. Plasmas 34620

[30] Fitzpatrick R 1998 Phys. Plasmas 53325

[31] Cole A and Fitzpatrick R 2006 Phys. Plasmas 13032503

[32] Heyn M, Ivanov I, Kasilov S, et al 2008 "Kinetic Estimate of the Shielding of Resonant Magnetic Field Perturbations by Plasma Rotation in DIII-D.” to be published in Nucl. Fusion

[33] Chirikov B V 1979 Phys. Reports 52265

[34] Rosenbluth M N, Sagdeev R Z, Taylor J B and Zaslavsky G M 1966 Nucl. Fusion 6 297 
[35] Rechester A B and Rosenbluth M N 1978 Phys. Rev. Lett. 4038

[36] Harvey R W, McCoy M G, Hsu J Y and Mirin A A 1981 Phys. Rev. Lett. 47102

[37] Rechester B, Rosenbluth M N and White R B 1979 Phys. Rev. Lett. 421247

[38] Krommes J A, Oberman C and Kleva R G 1983 J. Plasma Physics 3011

[39] Sauter O, Angioni C and Lin-Liu Y R 1999 Phys. Plasmas 62834

[40] Braginskii S I 1965 Reviews of Plasma Physics 1, Ed M A Leontovich, New York: Consultants Bureau, p 205

[41] Chodura R 1982 Phys. Fluids 251628

[42] Crotinger J A, Lodestro L L, Pearlstein L D, et al 1997 LLNL Report UCRL-ID126284, available from NTIS \#PB2005-102154

[43] Melnikov V K 1963 Trans. Moscow Math. Soc. 121

[44] Guckenheimer J and Holmes P 1983 Nonlinear Oscillations, Dynamical Systems, and Bifurcations of Vector Fields Applied Mathematical Sciences 42 New York: Springer-Verlag

[45] Wiggins S 2003 Introduction to Nonlinear Dynamical Systems and Chaos Texts in Applied Mathematics 2 New York: Springer-Verlag

[46] Jakubowski M W, Wingen A, Abdullaev S S, et al 2007 J. Nucl. Mater. 363-365 371

[47] Watkins J G, Evans T E, Joseph I, et al to be submitted to Nucl. Fusion Lett.

[48] Fenstermacher M E, Evans T E, Moyer R A, et al 2007 J. Nucl. Mater. 363-365 476

[49] Evans T E, DeGrassie J S, Jackson G L, et al 1987 J. Nucl. Mater. 145-146 812 
[50] Ghendrih Ph, Grossman A and Capes H 1996 Plasma Phys. Control. Fusion 381653

[51] Evans T E, Fenstermacher M E, Moyer R A, et al 2008 "RMP ELM Suppression in DIII-D Plasmas with ITER Similar Shapes and Collisionalities," to be published in Nucl. Fusion

[52] Stix T 1978 Nucl. Fusion 18353

[53] Tokar M Z, Evans T E, Gupta A, et al 2007 Phys. Rev. Lett. 9895001

[54] Nardon E, Bécoulet M, Huysmans G, et al 2007 J. Nucl. Mater. 363-365 1071

[55] Nardon E, Bécoulet M, Huysmans G, and Czarny O 2007 Phys. Plasmas 14092501

[56] Moyer R A, Burrell K H, Evans T E et al in Fusion Energy 2006 (Proc. 21st Int. Conf. Chengdu, 2006) (Vienna: IAEA) CD-ROM file EX/9-3 and http://www-naweb.iaea.org/napc/physics/FEC/FEC2006/html/index.htm 


\section{Figure Captions}

Figure 1. (a) Poincaré plot of the $\phi=0$ surface of section modeled for shot 123301 at $2170 \mathrm{~ms}$ for I-coil (3 kA-t), C-coil (12 kA-t) and intrinsic field errors. Field lines have been traced for a maximum of 200 toroidal transits and are colored from yellow to black by the number of toroidal turns before escape (200 to 1). Here, $\theta$ is not a straight-field line angle, but the standard poloidal angle defined from the magnetic axis. (b) $n=3$ Magnetic perturbation spectrum in straight field line coordinates for shot 122342 at $4650 \mathrm{~ms}$ for I-coil (3 kA-t), but without C-coil and field errors. Black and white line indicates resonant left-handed modes $m=-3 q$; white line indicates non-resonant righthanded $m=+3 q$ modes.

Figure 2. (a) For the I-coil spectrum alone [figure 1(b)], the Chirikov island overlap parameter rises above 1 near $\psi=0.95$. If we include the island widths, shown as horizontal bars, the transition to stochasticity takes place at $\psi=0.92$. (b) The quasi-linear field line diffusivity $\left(d_{f l}\right)$ and Kolmogorov length $\left(L_{K}\right)$ for the spectrum in figure 1(b) computed via equations (2) and (3).

Figure 3. Plasma parameters over the pedestal: (a) density and temperature, (b) parallel thermal diffusivity, (c) mean free path, (d) quasi-linear estimates of Kolmogorov length and thermal correlation lengths; $\exp =$ estimates based on measured H-mode profiles, $\operatorname{sim}=$ estimates based on simulation results for the I-coil $(3 \mathrm{kA}-\mathrm{t})$ perturbation. 
Figure 4. Simulation input: (a) density contours, (b) density versus poloidal flux on the outer midplane. Simulation results: (b) temperature profiles for unperturbed $(0 \mathrm{kA}-\mathrm{t})$ and perturbed (3 kA-t) cases.

Figure 5. Simulated temperatures at $\phi=180 \mathrm{deg}$. toroidal location: (a) unperturbed $T_{e}$, (b) unperturbed $T_{i}$, (c) perturbed $T_{e}$, and (d) perturbed $T_{i}$.

Figure 6. Quasi-linear estimates for the effective (a) parallel collisionality [equation (9)] and (b) electron and (c) ion thermal diffusivity for the plasma profiles in figure 3 . The best analytic estimates for the diffusivities, the minimum of the collisionless $(s t)$ and collisional $(R R)$ values, are marked by open symbols.

Figure 7. (a) The axisymmetric separatrix is formed from a one-parameter family of homoclinic orbits that both exit (red) and enter (blue) the X-point in the forward direction. (b) Under perturbation, the family of exiting field lines (red) no longer coincides with the family of entering field lines (blue). Escaping field lines are still confined by these invariant manifolds as they exit in the (c) forward and (d) backward toroidal directions. The Poincaré plots in (c) and (d) use all TRIP3D magnetic field sources [as in figure 1(a)] and are colored from red to blue by the number of toroidal transits until escape (200 to 1).

Figure 8 . The simulated electron temperature on the $0^{\circ}$ toroidal plane demonstrates that heat flux is efficiently guided along the invariant manifolds of the magnetic field lines that connect to the X-point: (a) forward stable manifold, (b) forward unstable manifold, (c) the homoclinic tangle. 
Figure 9. (a) Tangential X-point TV image of filtered $D_{\alpha}$ light displays three striations along the inner strike point on the right-hand side of the figure during I-coil operation (3 kA-t) in plasma discharge 123301. Part of the outer strike point can also be seen on the upper left-hand side. (b) The calculated first passage distribution of magnetic field lines as they strike the inner divertor target including C-coil, I-coil, and field errors contains significant $n=1$ and $n=3$ components. Shaded regions cover the field of view of the infrared camera centered on 60 (red) and the field of view of the tangential X-point camera centered on $180 \mathrm{deg}$. (blue) shown in (a). The direction of increasing major radius points from left to right in (a) and from bottom to top in (b).

Figure 10. Simulated heat flux deposition through the (a) inner and (b) outer strike points (ISP/OSP) due to the I-coil alone ( $3 \mathrm{kA}-\mathrm{t})$. The peak of the heat flux near $180 \mathrm{deg}$. (red solid) for both (c) ISP and (d) OSP is reduced as compared to the axisymmetric case (blue dash). The toroidal average of the perturbed profiles (black dot-dash) are wider than the axisymmetric cases indicating increased effective strike-point area. 
TRIP3D: \# forward for escape 1233012170 ms
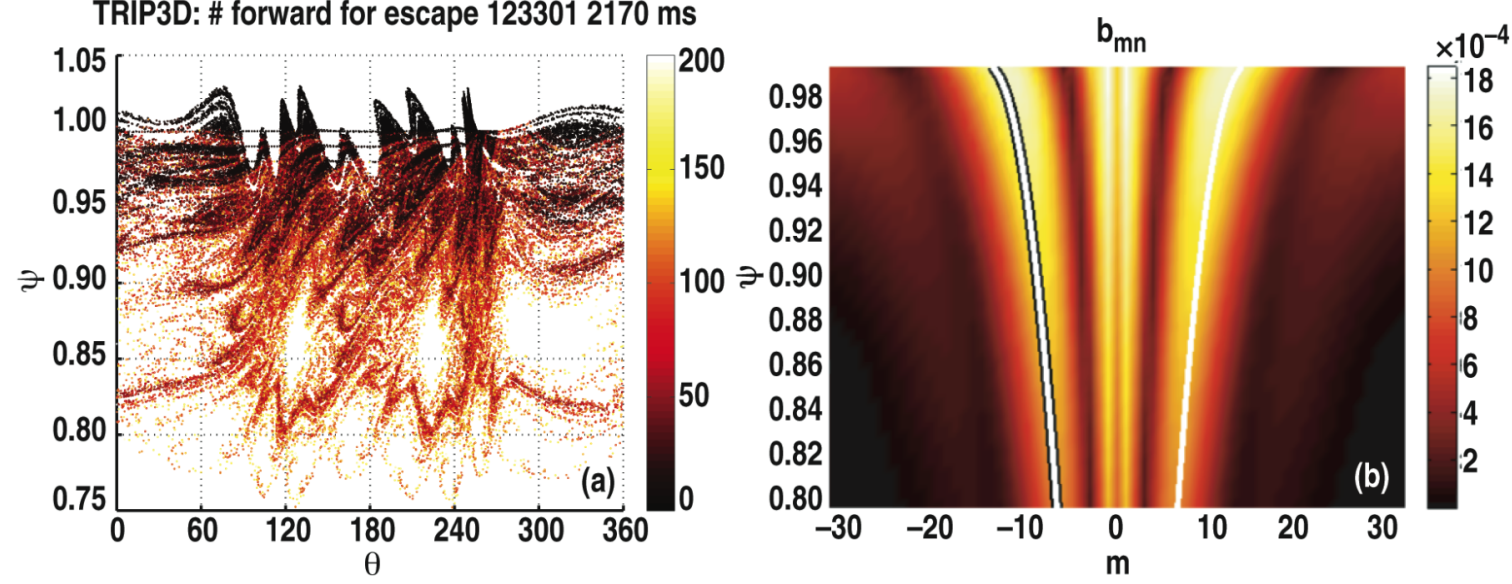

Joseph, I. Figure 1 

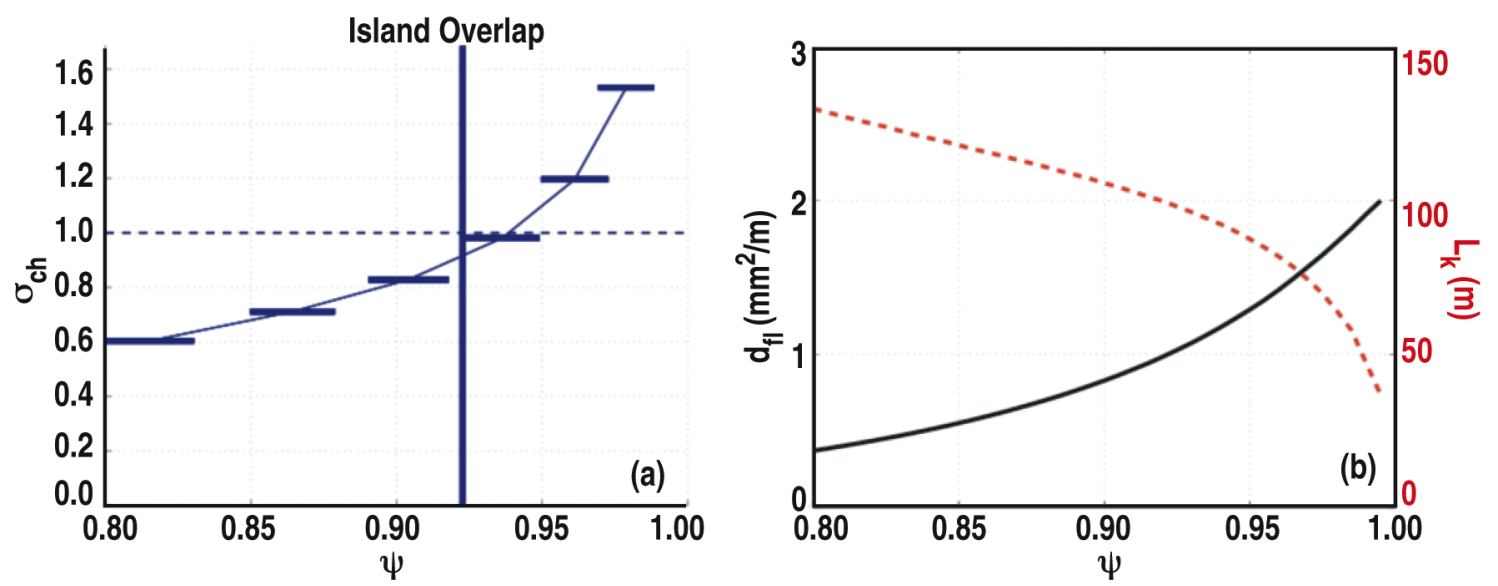

Joseph, I. Figure 2 

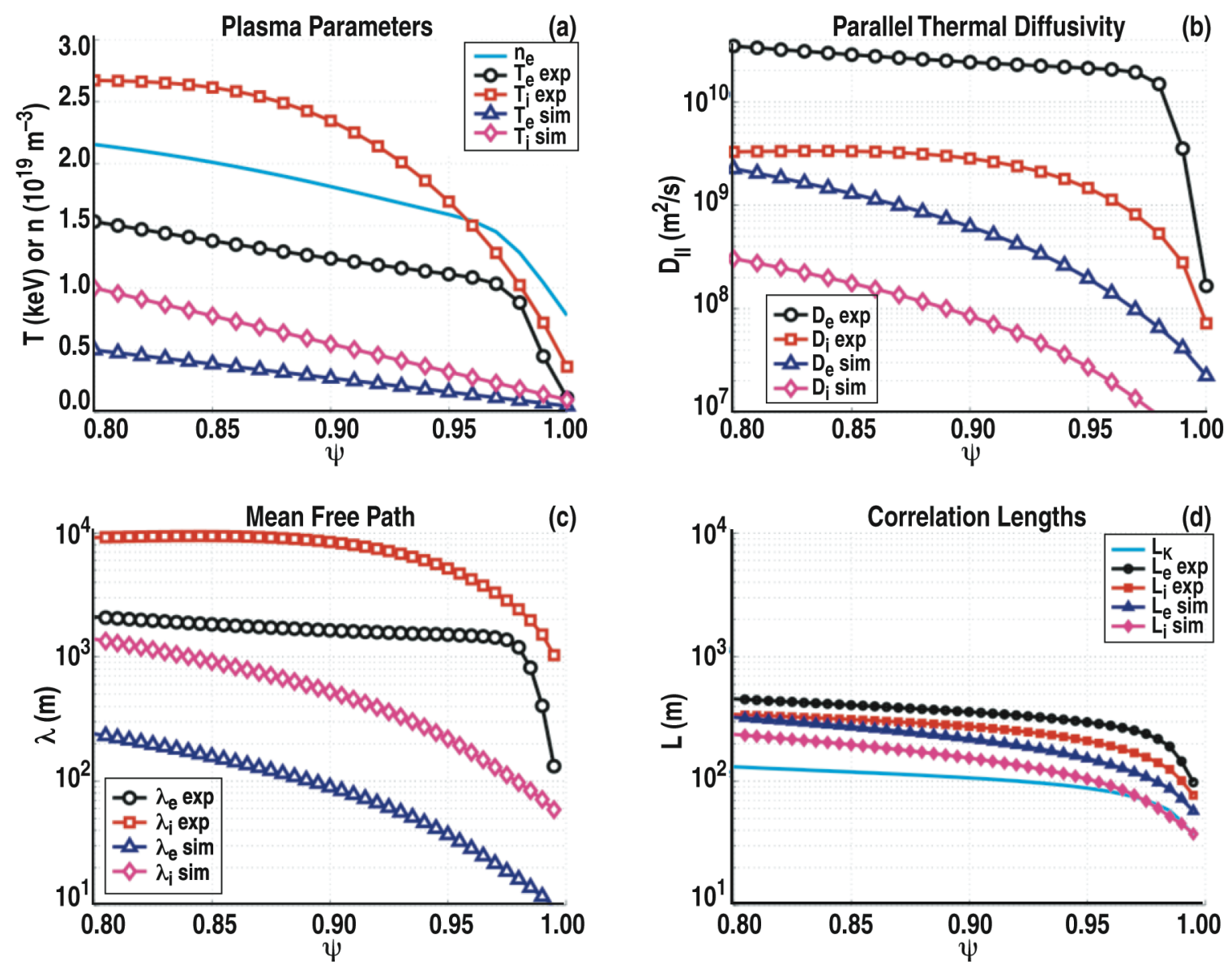

Joseph, I. Figure 3 

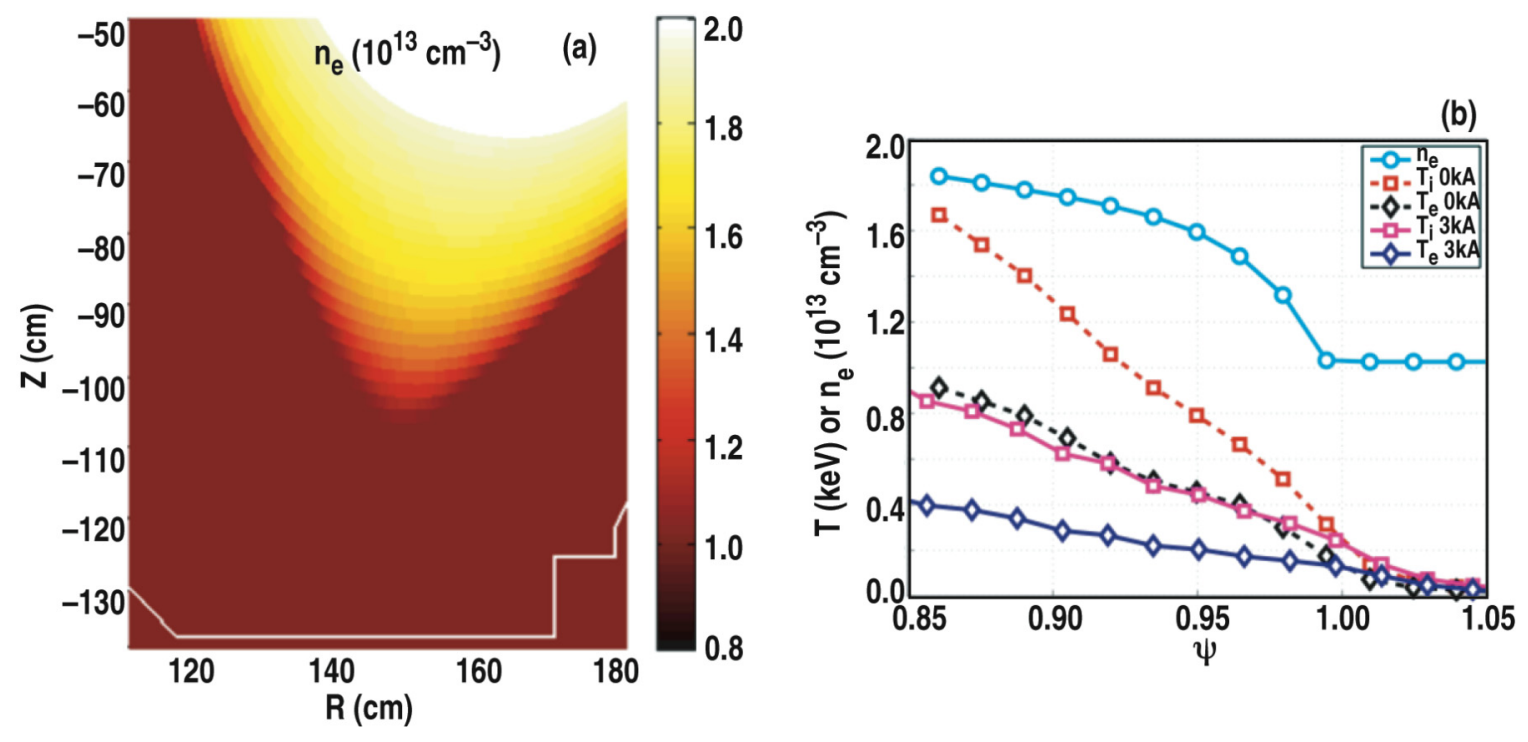

Joseph, I. Figure 4 

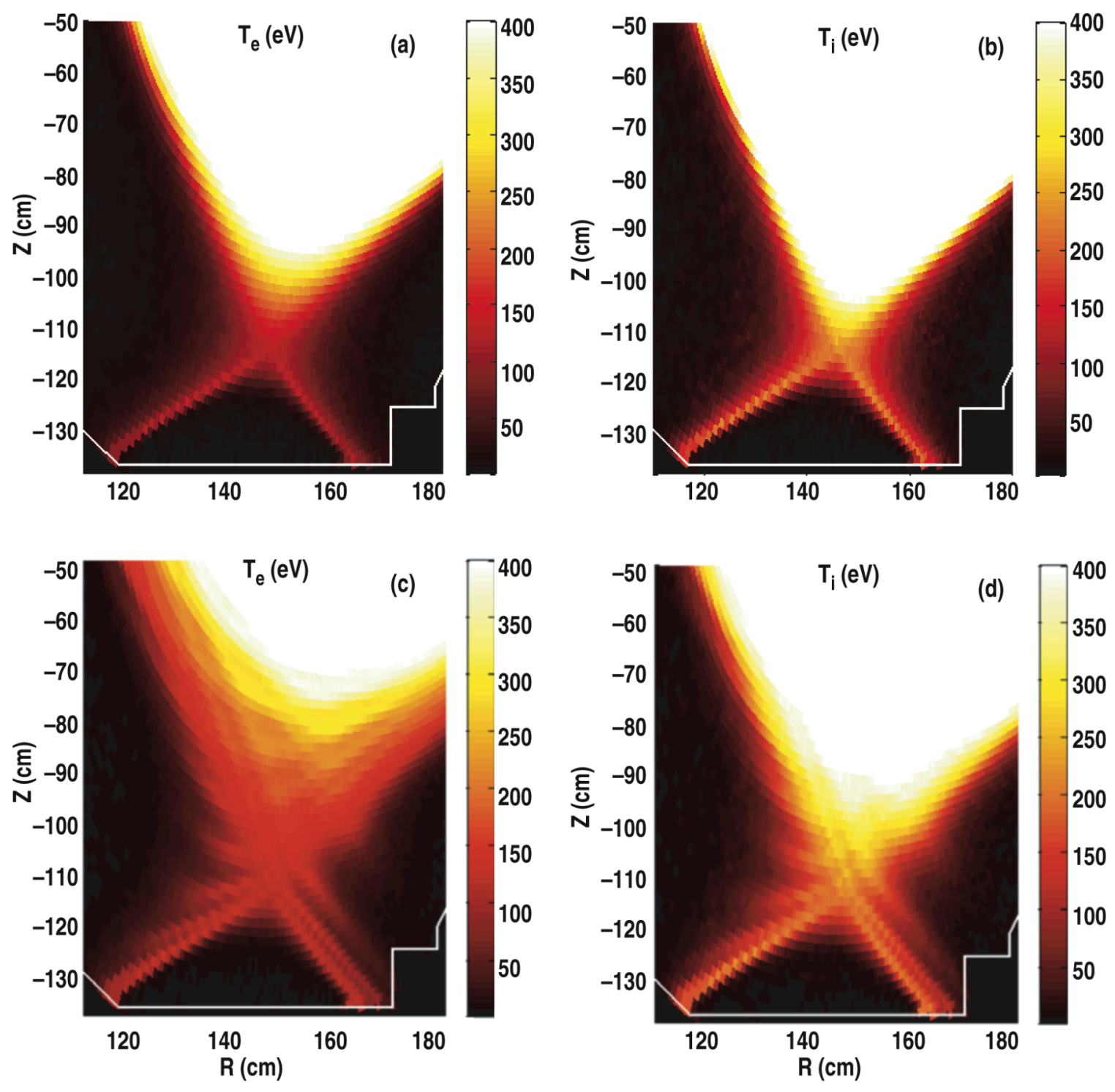

Joseph, I. Figure 5 

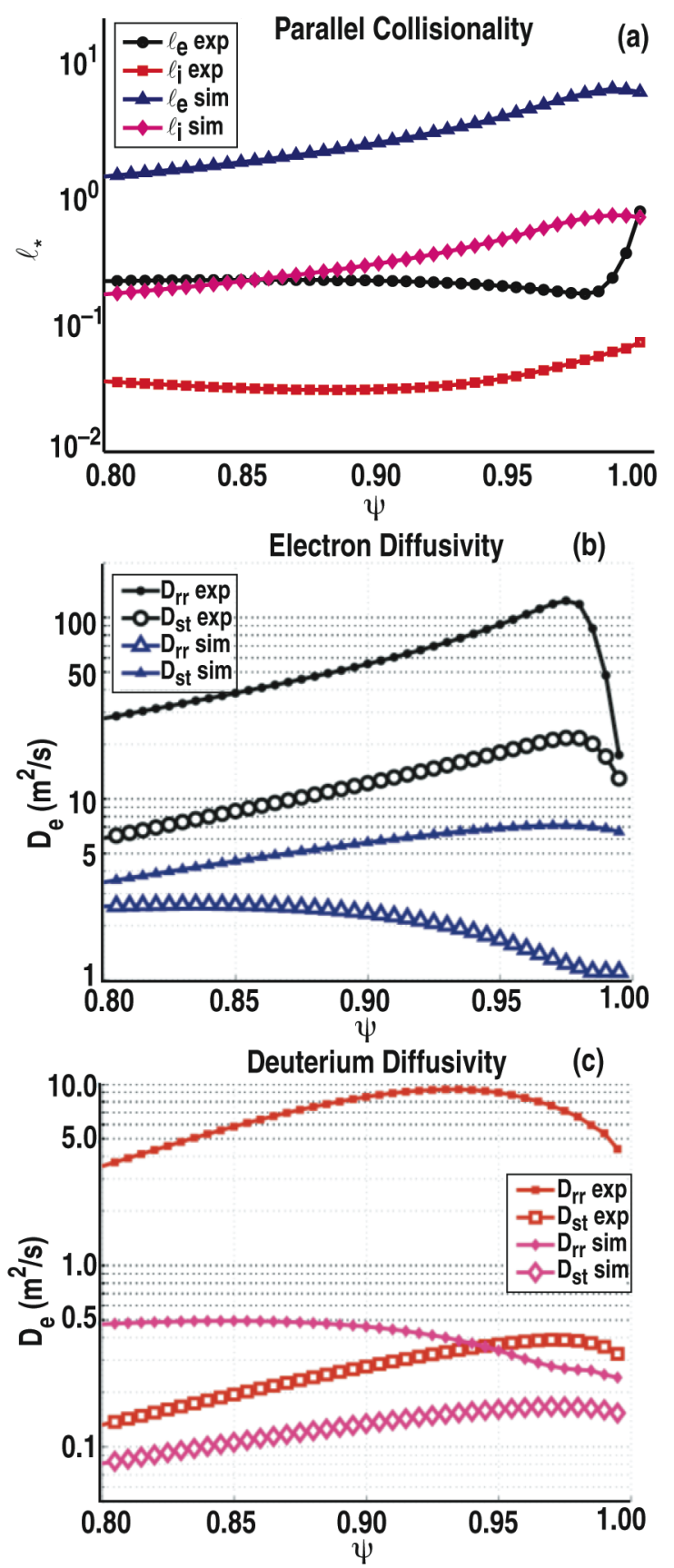

Joseph, I. Figure 6 

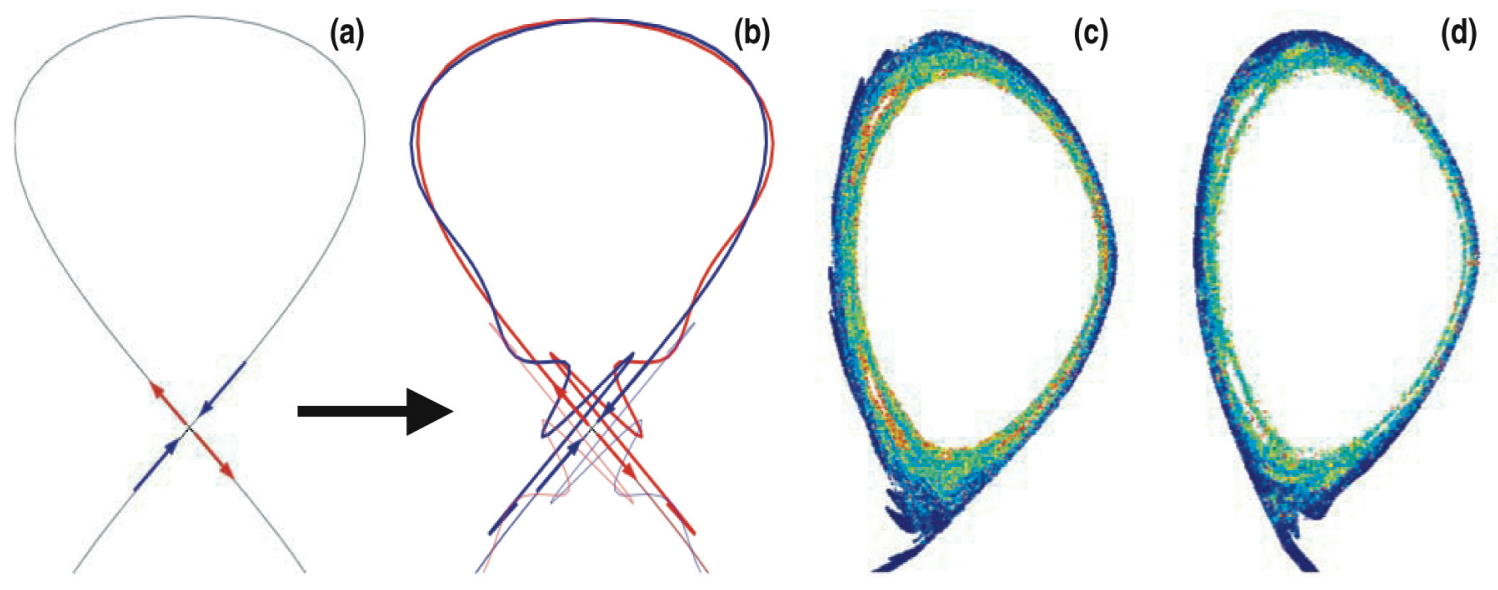

Joseph, I. Figure 7 

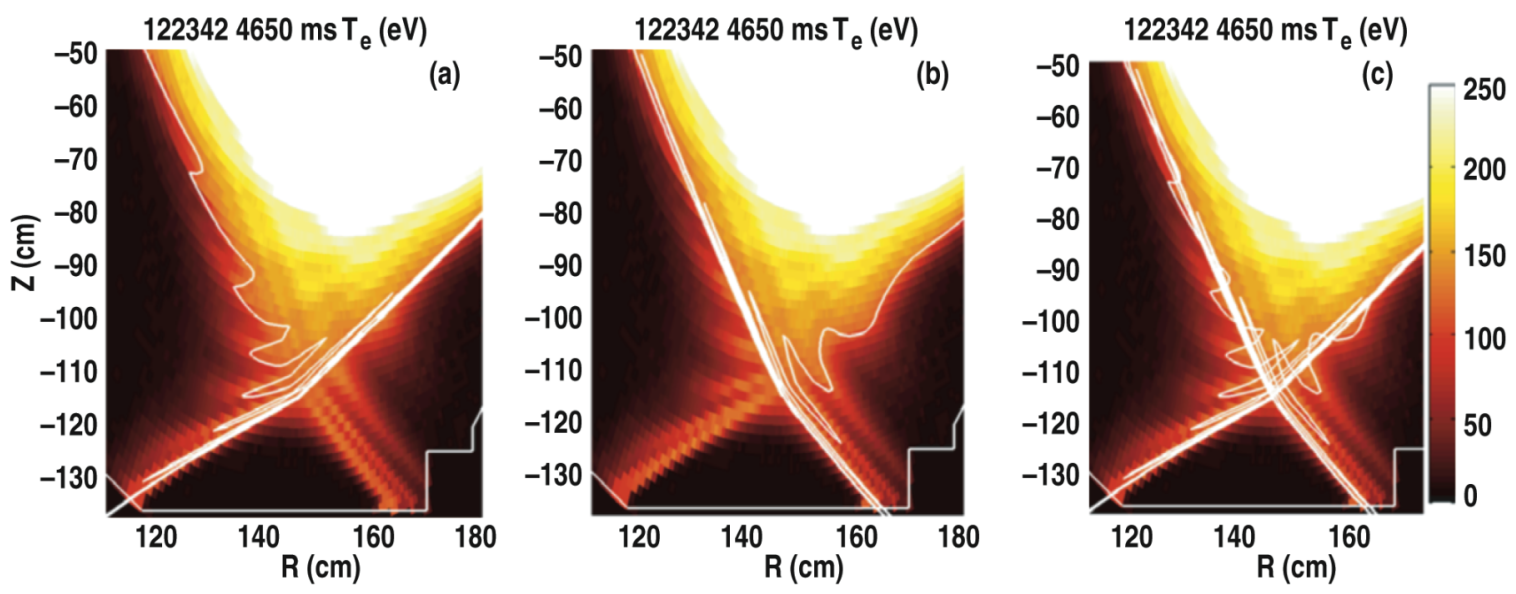

Joseph, I. Figure 8 
TRIP-3D: Inner Strike Point 123301

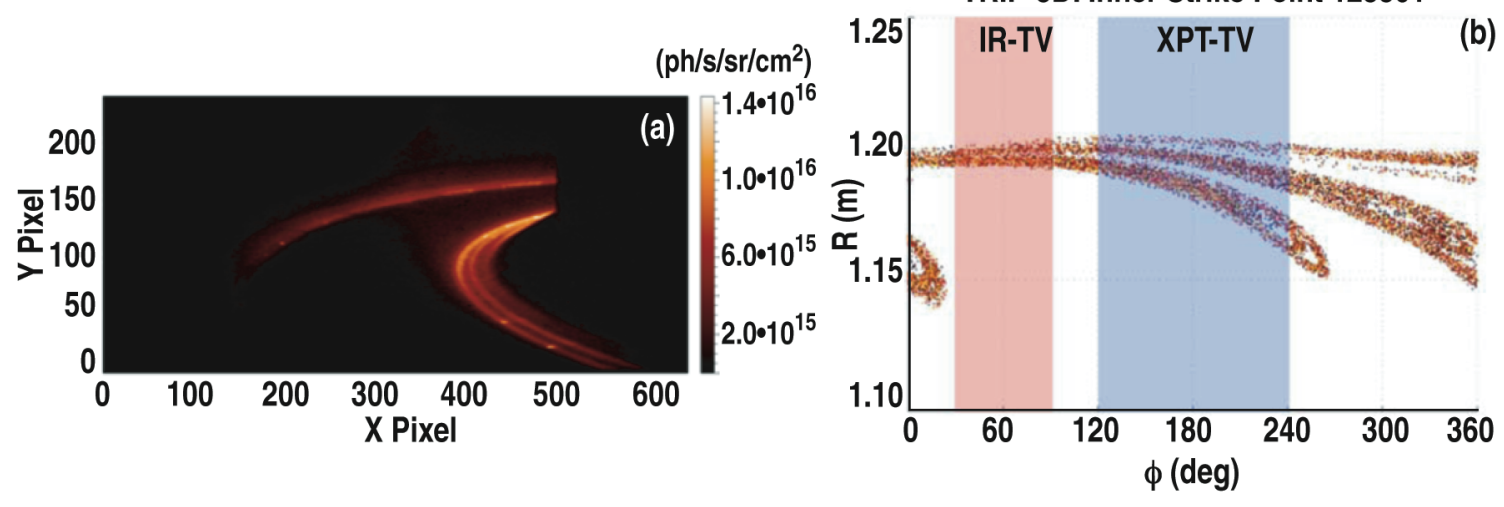

Joseph, I. Figure 9 

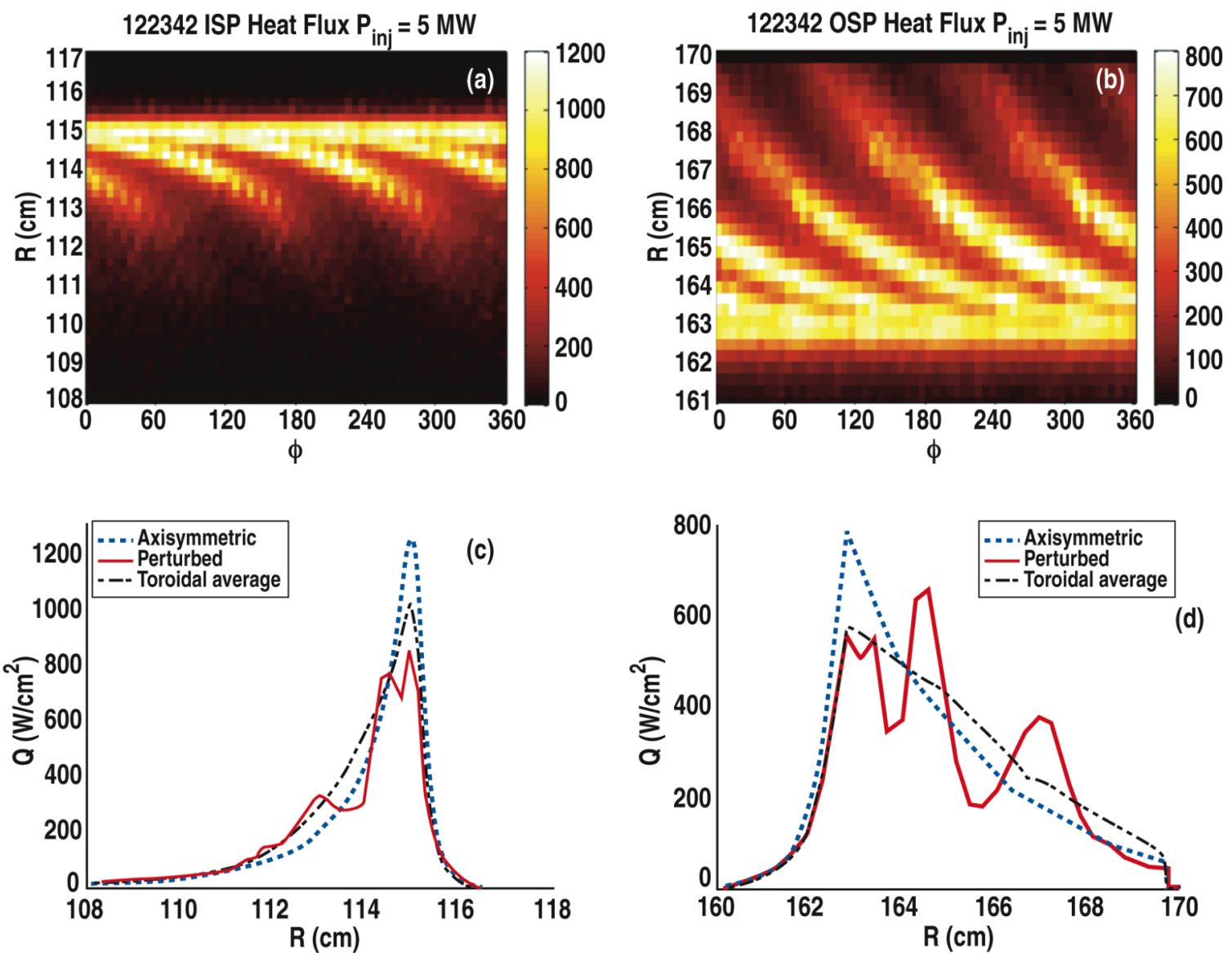

Joseph, I. Figure 10 